\title{
A simple non-linear model for internal friction in modified concrete
}

\author{
Daria Scerrato $^{1}$, Ivan Giorgio ${ }^{2,3}$, Angela Madeo ${ }^{4,2}$, \\ Ali Limam ${ }^{4}$ and Felix Darve ${ }^{5,2}$
}

September 21, 2013

\author{
${ }^{1}$ Dottorato in Meccanica Teorica ed Applicata, Università di Roma La \\ Sapienza, Italy \\ ${ }^{2}$ International Research Center for the Mathematics and Mechanics of \\ Complex Systems M\&MoCS, Cisterna di Latina, Italy \\ ${ }^{3}$ Dipartimento di Ingegneria Meccanica e Aerospaziale, \\ Università di Roma La Sapienza, Rome, Italy \\ ${ }^{4}$ Université de Lyon-INSA, 20 Av. Albert Einstein, Villeurbanne Cedex, \\ France \\ ${ }^{5}$ Institut Polytechnique de Grenoble, UJF-CNRS, Grenoble, France
}

\begin{abstract}
In this paper we consider a two-degrees-of-freedom, non-linear model aiming to describe internal friction phenomena which have been observed in some modified concrete specimens undergoing slow dynamic compression loads and having various amplitudes but never inducing large strains. The motivation for the theoretical effort presented here arose because of the experimental evidence described e.g. in $[94,24]$ in which dissipation loops for concrete-type materials are shown to have peculiar characteristics. Indeed, as (linear or nonlinear) viscoelastic models do not seem suitable to describe neither qualitatively nor quantitatively the measured dissipation loops, we propose to introduce a micro-mechanism of Coulombian internal dissipation associated to the relative motion of the lips of the micro-cracks present in the material. We finally present numerical simulations showing that the proposed model is suitable to describe some of the available experimental evidences. These numerical simulations motivate further developments of the considered model and supply a tool for the design of subsequent experimental campaigns.
\end{abstract}




\section{Introduction}

The standard models usually introduced to study the deformation of cyclically compressed specimens of modified (or not modified) concrete do not seem suitable to describe all the complex phenomena of internal dissipation which are experimentally observed, also in the range of small strains. Indeed, (see [94, 24]) the dissipation loops measured under cyclic loads with relatively small amplitude show very peculiar qualitative features (see Figs. 1 and 2):

- the two branches present different (varying) curvatures on the loading and unloading paths;

- the turning points seem to have the form of cusps ;

- the distance between the loading/unloading branches is varying in a nonlinear way with the applied compression loads.

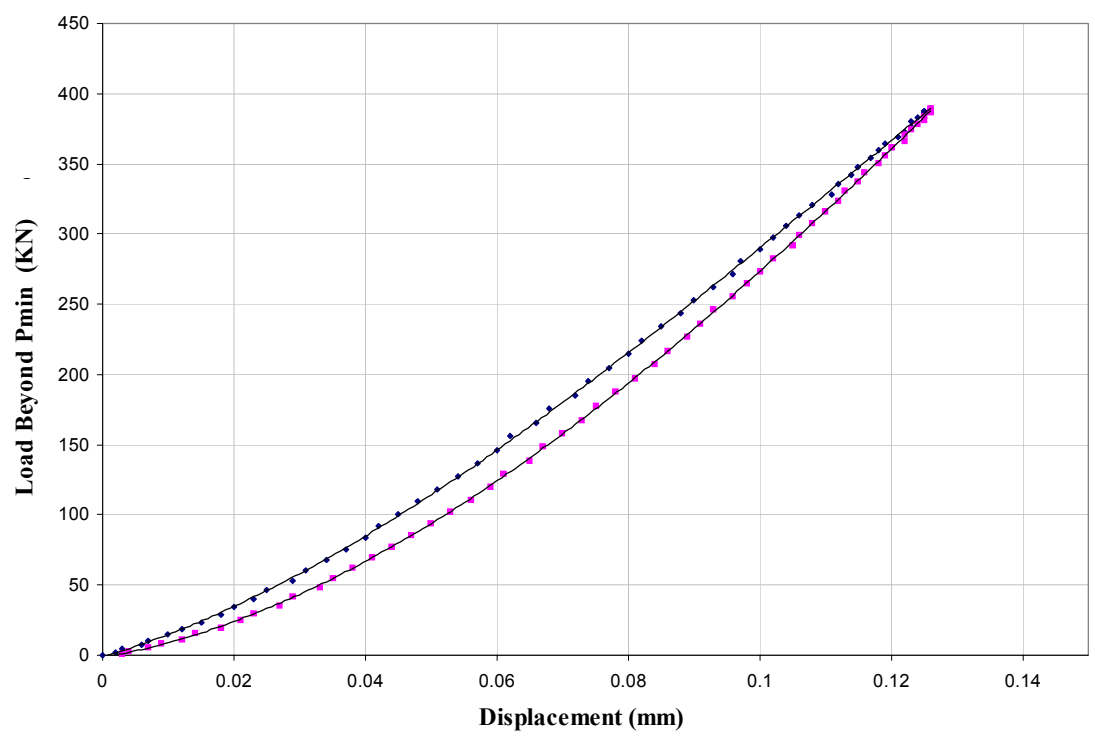

Figure 1: Example of measured dissipation loop in concrete-based material

Even when at macro-level the compression loads and deformations are relatively small, these experimental observations suggest that some non-linear elastic mechanisms at the micro-level together with a Coulombian dissipation mechanism related to micro-motions may explain the observed evidence. The discussion of the rigorous modeling and mathematical issues arising from the observed evidence is delicate and deserves attention, but is not the primary issue of this paper. In this work we will limit ourselves to show that a simple and efficient model is indeed conceivable to fit the targeted experimental evidences and to study its most relevant features. 


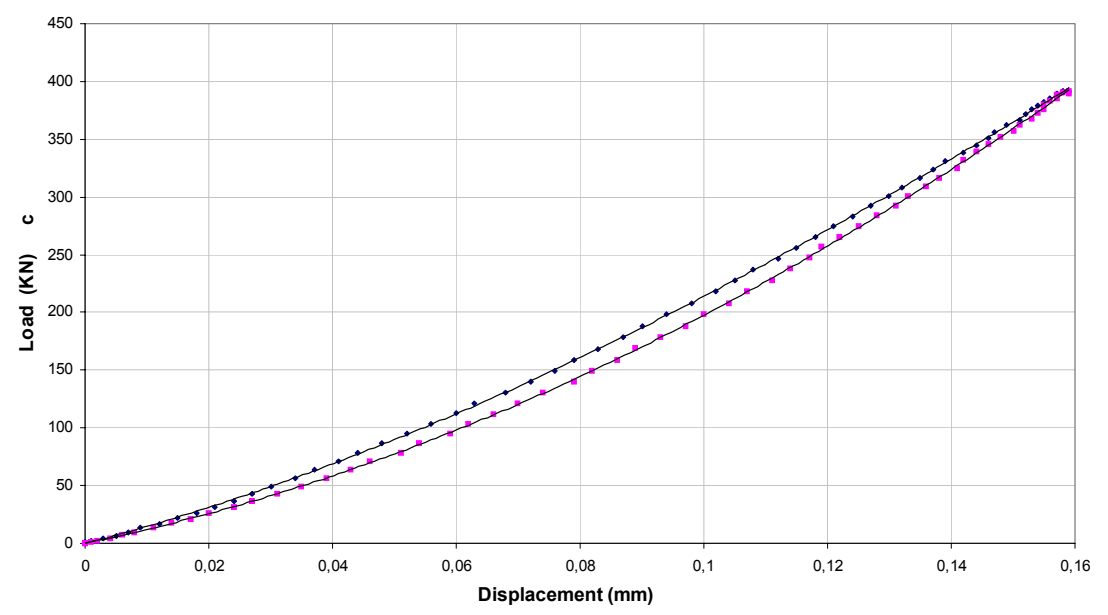

Figure 2: Example of measured dissipation loop in concrete-based material

\subsection{Models for internal dissipation in solids}

The problem of modelling internal dissipation in solids is very old and, for this reason, a huge literature concerning this subject is available. For clear reasons of consistency we limit ourselves to draft some considerations and to cite some among the relevant papers. It is indeed rather difficult to distinguish between the dissipated energy which participates to damage and plastic phenomena (even by producing new micro-cracks or enlarging the existing ones) and the dissipated energy which is associated to friction related to reversible internal relative motions. Interesting investigations which are meant to describe internal dissipation in presence of the damage progress are presented in $[131,22,117,66,65,88]$ where various mechanisms of damage and plasticity are taken into account, even at different length scales (see also [126, 130, 124, 125]). On the other hand, the classical works $[83,146]$ study the internal mechanisms of dissipation in solids when no relevant damage phenomena occur at the macroscopic level. In the same spirit are the papers [117, 66, 65, 54, 132, 147] where several models for dissipation of energy due to elastic deformation rate and relative motion of different parts of considered bodies are studied and applied in the case of metals or even concrete. Finally, two papers which accept a point of view very similar to ours are $[1,20]$ where the internal dissipation mechanism are clearly distinguished in two different types: one producing crack generation and growth, the second being related to reversible relative motion of micro-crack lips. In [1] micro-crack lips relative motion is described by means of a tensorial quantity, distinguished from plastic and elastic deformations. In some works (see e.g. [20]) the analysis is based directly on a finite-dimensional model to be used for numerical simulations: however, internal friction is there regarded as a phenomenon also driven by opening and closing of already existing cracks. Different works exist on the mathematical modeling of reversible damping ef- 
fects. In linear approaches, often stress-strain relations with integral kernels are used, while for geometrically nonlinear problems, the addition of rate-equations for some internal variables seems to be more useful, see e.g. $[113,112,114]$ for incorporation of grain boundary relaxation effects. The approach followed in the present paper follows the same spirit by means of the introduction of an internal variable $\varphi$ which is able to account for microscopic friction dissipation. These concepts can also be profitably extended to shells and plates (see e.g. $[116,115,148])$ and to rods (see e.g. $[18,19]$ ), where internal dissipation stabilizes the model, leading to robust numerical algorithms and profound theoretical results.

\subsection{Continua with microstructure}

In this paper we choose to introduce an extra kinematical field $\varphi$, in addition to the standard macroscopic displacement, to describe the relative displacement of superimposed lips of the microscopic cracks which are known to be present in concrete materials. The fact of introducing extended kinematics to study materials with microstructure was developed by many authors in the decades 1950-1980 (see e.g. [64, 60, 63, 61, 62, 64, 60, 63, 61, 62, 79, 80, 78, $77,103,104,105,136,137,79,78,77,103,104,105,136,137])$ to study deformation, damage and internal friction phenomena occurring in deformable bodies which, at a suitable micro-level, may show strong inhomogeneities in geometrical and mechanical properties. Models of continua with enriched kinematics are known as micromorphic models and originated with the works of Mindlin [103] and Eringen [64]. Such micromorphic models can also be seen as a way to obtain, as suitable limit cases, other very common generalized continuum theories which are known as second gradient theories (see e.g. [74, 75]). These latter, have the same kinematics as classical Cauchy continuum theories (only displacement field), but the microstructure of the continuum is accounted for by suitable length-scales associated to the fact that the strain energy density is assumed to depend on the second gradient of displacement. Various micromorphic and higher-gradient theories have been re-proposed in the last years to study different kinds of physical systems with microstructure (see e.g. $[43,70,72,73,71,84,106,109,108,107,120,138,139,140,14,15])$. Recently, a relaxed micromorphic model have been proposed in $[76,111]$ which allows to account for possible micro-heterogeneities with a considerably reduced number of constitutive parameters with respect to classical micromorphic models. We can summarize by saying that micromorphic models are able to catch the complexity of the coupling phenomena between micro- and macro-motions. We claim that these micro-macro coupling phenomena actually dominate dissipation in solids constituted by grains bonded with viscoelastic cement. As the internal frictional phenomena which we have in mind obviously can also occur in beams and shells the concept of additional microstructural or internal variable we discuss here may be adapted to many different cases as for instance the continuum models treated in the papers $[5,9,8,6,4,56,57,58,59,7]$ for shells with surface stresses or micro-polar extra kinematical descriptors and $[3,4,10]$ ) 
for continua showing viscoelastic behaviour. In future investigations it could be needed to conceive homogenization procedures aiming to deduce the most suitable macroscopic models for describing the phenomena we have in mind (see e.g. [2] for a homogenization process leading to second gradient materials) and which seem to be associated to the proposed model of continuum with microstructure. This would allow to set up a micro-macro identification procedure for the determination of macroscopic constitutive parameters characterizing the mechanical behaviour of modified concrete starting from its microscopic internal structure.

In this paper we consider a continuum model with microstructure aiming to describe some of the dissipation phenomena occurring in deformable bodies which, at a micro-level, are constituted by a compact matrix presenting a uniform and isotropic distribution of penny-shaped micro-cracks. We are aware of the fact that this last assumption is rather simplified: not only micro-cracks are not in general isotropically and homogeneously distributed in the reference configuration, but even if they are so, they will lose these properties in the current configuration. Therefore, one should expect that for a careful and detailed modelling of the phenomena that we have in mind one should introduce some tensor fields (see e.g. [1]) in order to describe the evolution of the state of micro-cracks inside the Representative Elementary Volume (REV). This delicate point is not addressed in the present paper and we will limit ourselves to consider a simple scalar field $\varphi$ whose evolution describes, in an approximate and averaged way, the total amount of relative displacement of crack lips present in the considered REV. Indeed, it will be assumed that the number of micro-cracks, their geometrical and mechanical properties do not change in considered dynamic phenomena. In other words we assume that damage is not progressing: actually the dissipative phenomena which we want to describe are related to reversible contact phenomena at crack lips (see e.g. [89] and references there cited).

\subsection{Methods for reducing the degrees of freedom in con- sidered models}

The concrete specimens tested in the experimental campaigns described in [94] and the behavior of which we want to reproduce, are three-dimensional specimens. Therefore, the suitable model to be introduced is a model (eventually non-linear) of a three-dimensional continuum. However, to simplify the used model one can think to follow the method pioneered by Saint-Venant (for some generalizations of the classical problem named after him and the discussion of the central idea of his method see e.g.[16, 49, 40,12] and the references there cited). This method allows for the reduction of the complexity of the problem of

determining the deformation of a specimen the shape of which is cylindrical in its reference configuration. More particularly, the problem of solving a system of PDEs is reduced into a simpler one: solving a system of ODEs.

In the present paper we assume that

- considered cylinders are deformed only in the Saint-Venant compression (with Poisson effect) mode; 
- frequency of the forcing load is much smaller than the eigenfrequencies of the cylinders;

- the distribution of micro-cracks is uniform so that one scalar quantity is sufficient to describe the internal micro-motion in the cylinder;

- the present imperfections do not cause or do not influence in any way (neither global nor local) instabilities and in particular do not change microstructure evolution (the literature in the field is immense, the reader is referred e.g. to $[90,85]$ and references there cited).

Once having assumed the previous hypotheses the problem to be solved consists then in

- looking for the evolution equation for the newly introduced kinematical parameter $\varphi$ describing micro-displacement with Coulombian dissipation;

- determining the modifications to be introduced in standard evolution equations for the displacement field;

- determining the coupling terms between these equations.

Of course, in order to get the reduced model one could conceive a more or less mathematically rigorous process starting from a Cauchy model at a given small length scale for the material inside a REV, in which the boundary conditions at crack lips are chosen to include Coulombian friction. We do not address such a delicate analysis in this paper and we will instead formulate a macroscopic model which shows the qualitative behavior which is expected on the basis of appropriate phenomenological considerations. In fact, we present a target theory to which such a homogenization process should try to arrive. It is clear that the constitutive parameters appearing in the phenomenological theory which we develop can be determined only by means of the comparison of suitable numerical simulations with corresponding experimental evidence: in this paper we present suitable parametric studies of the proposed model in order to be able to subsequently tune these parameters to direct the design of a measurements campaign.

The model presented here may have a wide applicability range: however it is tailored with a view towards a precise application which is of interest in structural and civil engineering. Actually, our attention is focused, in particular, on the description of mechanical behavior of concrete-like materials with enhanced internal frictional dissipation. This enhancement is obtained by changing the recipe of the concrete mix by adding suitable inert additives, whose grains have the size needed to fill crack voids or to improve friction contact of crack lips (see e.g. $[94,117,24]$ and references there cited). The numerical simulations which are presented in this paper needed to be calibrated carefully because of the strong nonlinearities they present and because of the singular nature of ODEs usually associated to Coulomb friction. These points are not addressed in the present investigation (in this aspect, and also for what concerns the modelling difficulties, the model here proposed resembles closely the one studied in 
[46]). The numerical results obtained show that the proposed model is suitable to describe some of the experimental evidences discussed in [94]. The main peculiarities of the proposed model can be resumed as follows:

- nonlinearities appear only in terms of the parameter $\varphi$ and its time derivative,

- Coulombian dissipation mechanism plays a role only in the evolution equation for $\varphi$,

- dissipation cycles in the force-displacement plane show a variety of qualitative behaviors depending on the choice of constitutive parameters which is wide enough to encompass the structure which has been observed e.g. in [96] and reproduced in Figs. 1 and 2.

\subsection{Some considerations about future perspectives and limits of the presented model}

The system of ODEs we consider, presents non-trivial mathematical difficulties which need to be studied, in order to assess their possible use in the considered applications. This mathematical study is preliminary to the formulation of a suitable variational principle to be developed in order to supply the governing equations of systems which are more general than the one considered here. Moreover, the role of the micro-displacement parameter $\varphi$, as conceived in this paper, will need to be further specified. More specifically: one may ask himself if it is possible to regard such a parameter as an internal variable (as done in very similar models, see e.g. [41, 42, 44, 45] and references there cited) or if it should be regarded as a truly micro-structural parameter (as done e.g. in $[136,137$, $78,77,103,108,107,135,49]$ and references there cited). The mathematical relevance of such a question is not negligible. Indeed, in the first case the deformation energy is assumed to depend on $\varphi$ only, while in the second one also a dependence on $\nabla \varphi$ will appear. As a consequence, the evolution equations for $\varphi$ in the first case will not involve partial derivatives, which will instead be present in the second case. A related problem concerns the highest order of the derivatives of the placement field which one will need to introduce in deformation energy to obtain the searched generalized model: it is indeed known (see e.g. [2] and references there cited) that high contrast in the mechanical properties at the micro-level (which surely is present in the physical system considered here) requires the introduction of higher order continua (see e.g. [50, 43, 70, 72, 73, 71, $97,110,134]$ and references there cited). In this paper we are assuming that the population of cracks present in considered specimens is not varying in number and is not changing its mechanical and geometrical properties because of the applied loads. In other words we assume that the level of damage (as modeled in different ways see e.g. [1, 3, 13, 20, 22, 23, 30, 33, 35, 32, 31] and references there cited) at microlevel is not changing during considered loading/unloading processes. Indeed, the experimental evidence presented in [94] shows that one has not a degradation in the mechanical properties of considered specimens 
when the intensity of the applied loads is below a precise threshold and if the duration of loading is limited in time. This means that dissipation occurs at crack lips without relevant abrasion (see e.g. [89] and references there cited), without any plastic phenomenon (see e.g. [33, 35, 34, 38, 37] and references there cited) and that there is no formation of new cracks (as described in [82] and the literature stemming from it).

We will not assume in this paper an explicit dependence of the strain energy density on the space gradient of $\varphi$. This means that we are assuming that the micro-motion inside one REV does not depend on the microscopic motions inside the contiguous ones. This is equivalent to say that we do not account for the possibility of describing, at a macroscopic scale, the presence of high gradients of microscopic relative displacements. This hypothesis is quite sensible since no localization phenomena are observed in the performed mechanical tests. The model which is presented here has been tailored to be used for modified concrete. However, one should consider that many possible applications can be imagined for it. Actually, internal friction is a phenomenon which plays a relevant role in different contexts: for instance in the flow in porous media (see e.g. [50, 46, $69,51,97,106,123,122,133,134]$ and references there cited) where internal friction competes with Darcy or Brinkman dissipation or in growing tissues (as described using micro-structured continuum models see e.g. [67, 68, 87, 96, 95]) where internal friction seem to play a non-negligible role in those mechanosensing phenomena controlling tissue synthesis.

It has to be finally remarked that the modifications of concrete by means of suitable inert additives proposed in [94] are founded on some precise theoretical considerations (see e.g. [117]) which need to be further developed. Actually, the idea is the following: the concrete recipe is modified in order to increase the dissipation capability without favoring the onset of plastic deformation or crack formation and growth. In order to develop this idea an important theoretical effort is required: one has to adapt the methods used in the theory of structural modification (see e.g. [28, 29, 27, 36] and references there cited) or in the design of smart materials and structures (see e.g. [11, 47, 48, 98, 99, 100, 121, 144, 143] and references there cited). In the first type of theories the concept which is exploited is the following: one adds to the system some coupled subsystems in which the energy preferably flows and in which it remains trapped until it is somehow dissipated. We remark that this effect can be obtained by using only linear phenomena (see [28]) and that its analysis is based on the concept of internal or micro-structural evolutionary variables whose evolution equations are suitably designed. This is also the concept on which the second of the aforementioned research streams is based: the coupled systems are designed in order to internally resonate with the principal system and the damping elements are tailored to optimize energy dissipation.

In conclusion, the change of concrete recipe which is envisaged here should forecast the formation of suitable slave microstructures

- whose motion is activated by macro-deformation

- whose presence do not modify the mechanical strength of the modified 
concrete

- whose damping capabilities reduce (or do not alter) the damage progress in presence of cyclic load.

One of the main conclutions to which the present paper leads is that the modelling tool which seems more suitable in this context is a microstructure field theory coupled with standard methods of optimization and control. More precisely, once having introduced a new scalar kinematical parameter $\varphi$, which is intended to measure the overall relative displacement of micro-cracks lips inside a Representative Elementary Volume (REV) of considered specimens, we postulate, on the basis of reasonable simplifying assumptions, some coupled Ordinary Differential Equations governing both macroscopic strains and micro-deformations of internal micro-cracks. We aim to describe i) linear and nonlinear deformation phenomena occurring because of aforementioned relative displacement ii) coupling phenomena between macro-motion and micro-motion and iii) Coulomb dissipation phenomena occurring at crack lips. The presented model assumes no crack growth or crack production but simply the existence of a reversible (although dissipative) internal micro-motion due to the presence, in the reference configuration, of micro-cracks.

\section{Modelling}

The model for concrete-based materials which we want to introduce is a very particular case of continuum with microstructure (see e.g.[60, 103]). Our final aim is to investigate the mechanism of internal dissipation due to Coulombian friction. Many authors examine the different mechanisms of internal dissipation in brittle materials such as concrete, see e.g. for more details [88], [20]. In particular, we want to focus our attention on dissipation in materials (as concrete) which present voids typically characterized by a shape as a coin (penny-shaped cracks), and materials (as modified concrete e.g. [94, 24]) containing microscopic components such as micro-filler used to improve damping performances without compromising mechanical strength. An additional and independent microstructural kinematical variable $\varphi$ is newly introduced to take into account the interaction between the opposite faces of voids (micro-cracks) when they come in contact and slide one with respect to the other because of the externally applied load. We assume that the deformation energy of the considered medium is characterized by a volume strain energy density $\Psi$ which depends on the basic kinematic descriptors, i.e. the classical displacement field $\mathbf{u}$ and the new scalar field $\varphi$ which represents the relative displacement of two superimposed lips of considered micro-cracks. In particular, we set

$$
\Psi(E, \varphi)=\frac{1}{2}\left(2 \mu \mathbf{E} \cdot \mathbf{E}+\lambda(\operatorname{tr} \mathbf{E})^{2}\right)+\frac{1}{2} k_{1} \varphi^{2}+\frac{1}{3} k_{2} \varphi^{3}+\frac{1}{4} k_{3} \varphi^{4}+\alpha \varphi \sqrt{I_{2}^{(d)}}
$$

where $\lambda$ and $\mu$ are the Lamé parameters for linearly elastic isotropic materials, $\mathbf{E}=\left(\nabla \mathbf{u}+\nabla \mathbf{u}^{T}\right) / 2$ is the linearized Green-Lagrange deformation tensor and 
the scalar $I_{2}^{(d)}$ is the second invariant of the deviatoric strain tensor skew $\mathbf{E}=$ $\mathbf{E}-\frac{1}{3} \operatorname{tr} \mathbf{E} \mathbb{1}$ defined as

$$
I_{2}^{(d)}=\frac{1}{2} \operatorname{tr}(\text { skew } \mathbf{E} \text { skew } \mathbf{E}),
$$

On the other hand, the kinetic energy density of the considered system is defined by

$$
\mathcal{K}=\frac{1}{2} \rho \dot{\mathbf{u}}^{2}+\frac{1}{2} \rho_{\varphi} \dot{\varphi}^{2}
$$

where $\rho$ is the mass density of bulk material and the $\rho_{\varphi}$ is an effective macroscopic mass density linked to the micro-structural variable $\varphi$. It can be checked that, since the micro-strain field $\varphi$ is assumed to have the dimensions of a length, the units of the parameter $\rho_{\varphi}$ are the same as the units of the macroscopic bulk mass density $\rho$. Since the dissipation is not negligible, the governing equations of the considered medium do not have a variational structure, but they possess a quasi-variational structure. In this context we introduce a Rayleigh potential $\mathcal{R}$, which is aimed to describe Coulomb-type friction dissipation, in the form

$$
\mathcal{R}=\zeta \operatorname{tr} \mathbf{E}\left(\frac{\log (\operatorname{Cosh}(\eta \dot{\varphi}))}{\eta}\right)
$$

where $\zeta$ and $\eta$ are constitutive constants. We can notice that, in particular, the constant $\eta$ can be seen as the inverse of a characteristic velocity associated to microscopic motions, while the constant $\zeta$ directly accounts for dissipation due to microscopic frictional sliding. Few words need to be spent here: actually in the literature Coulomb friction force is usually modeled by the introduction of the function signum whose argument is the velocity of the kinematical quantity on which friction forces are acting. This function, when appearing in differential equations, is a source of strong singularities and numerical or chaotic instabilities (whose regularization and study can be performed using the methods described in the literature (see e.g. [91, 92, 102, 93] and references therein). Also basing ourselves on physical considerations, we propose to regularize the function signum with an hyperbolic tangent modulated with an amplitude $\zeta$ (giving the maximum of friction force which may be exerted) and with a suitably chosen slope given by $\eta$ (triggering the range of velocity where friction force is an increasing function of the velocity). This is equivalent to state that we assume a Navier-Stokes type of dissipation for low micro-velocities: this physical assumption, although reasonable, needs to be justified with more detailed analyses and can also be related to bifurcation and instability phenomena which may occur in modified concrete (see e.g. [85] and references therein).

In conclusion, the virtual work due to internal dissipation can be written as

$$
\delta W^{(\text {Diss })}(\mathbf{E}, \varphi, \dot{\varphi})=\int_{0}^{T} \int_{V}\left(\frac{\partial \mathcal{R}}{\partial \dot{\varphi}} \delta \varphi\right) \mathrm{d} V=\int_{0}^{T} \int_{V} \zeta \operatorname{tr} \mathbf{E} \tanh (\eta \dot{\varphi}) \delta \varphi \mathrm{d} V,
$$

where $V$ is the volume which the specimen occupies in its reference configuration and $[0, T]$ is the time interval during which we observe the motion of the 
specimen. We can write the governing equations in weak form of the considered concrete-based material as

$$
-\delta W^{(\text {Elast })}+\delta W^{(\text {Iner })}=-\delta W^{(\text {Diss })}+\delta W^{(E x t)}
$$

in which

$$
\begin{gathered}
\delta W^{(\text {Elast })}=\int_{0}^{T} \int_{V} \delta \Psi \mathrm{d} V, \quad \delta W^{(\text {Iner })}=\int_{0}^{T} \int_{V} \delta \mathcal{K} \mathrm{d} V \\
\delta W^{(E x t)}=\int_{0}^{T} \int_{V} \mathbf{b}^{e x t} \cdot \delta \mathbf{u} \mathrm{d} V
\end{gathered}
$$

with the expressions for the strain energy and kinetic energy densities given by Eqs. (1) and (2) respectively and where $\mathbf{b}^{\text {ext }}$ are the bulk externally applied forces.

\subsection{Simplified equations of motion based on Saint-Venant theory for the case of simple compression}

We use from here on the fact that the experiments which are targeted in this work are simple compressions of cylindrical concrete specimens, so that we assume that Saint-Venant theory for simple compression can be applied. In this way, we are able to deduce the simplified equations in strong form directly from expression (4). To do so, we start recalling that, in the case of axial compression along the $x_{3}$ axis of a Saint-Venant cylinder, one has

$$
\mathbf{E}=\left(\begin{array}{ccc}
-\nu \varepsilon & 0 & 0 \\
0 & -\nu \varepsilon & 0 \\
0 & 0 & \varepsilon
\end{array}\right), \quad \mathbf{u}=\left(\begin{array}{c}
-\nu \varepsilon x_{1} \\
-\nu \varepsilon x_{2} \\
\varepsilon x_{3}
\end{array}\right)
$$

where we set $\varepsilon=E_{33}=u_{3,3}, \nu$ is the Poisson coefficient and $x_{1}, x_{2}$ and $x_{3}$ are the Lagrangian coordinates (in a given reference frame with origin on the axis of the cylinder) of the material points constituting the considered specimen. We also explicitly remark that another assumption of the Saint-Venant model is that the field $\varepsilon$ does not depend on $x_{1}, x_{2}, x_{3}$, but only, eventually, on time. We make the same assumption for the micro-displacement field $\varphi$. With these simplifying assumptions, it can be checked that in the considered particular case, integrating by parts in time, considering isochronous motions and arbitrary variations $\delta \varepsilon$ and $\delta \varphi$ the principle of virtual powers (4) implies

$$
\begin{aligned}
& M \ddot{\varepsilon}+K \varepsilon+\tilde{\alpha} \varphi=f_{0}+f_{1} \sin (\omega t), \\
& m_{\varphi} \ddot{\varphi}+\tilde{k}_{1} \varphi+\tilde{k}_{2} \varphi^{2}+\tilde{k}_{3} \varphi^{3}+\tilde{\alpha} \varepsilon-\tilde{\zeta} \tanh (\eta \dot{\varphi}) \varepsilon=0,
\end{aligned}
$$


where

$$
\begin{gathered}
M=\int_{V} \rho\left(\nu^{2}\left(x_{1}^{2}+x_{2}^{2}\right)+x_{3}^{2}\right) d V, \quad m_{\varphi}=\int_{V} \rho_{\varphi} d V \\
K=\int_{V}\left(\lambda+2 \mu+4(\lambda+\mu) \nu^{2}-4 \lambda \nu\right) d V, \quad \tilde{\alpha}=\int_{V}(\sqrt{3} / 3(1+\nu) \alpha) d V \\
\tilde{k}_{1}=\int_{V} k_{1} d V, \quad \tilde{k}_{2}=\int_{V} k_{2} d V, \quad \tilde{k}_{3}=\int_{V} k_{3} d V, \quad \tilde{\zeta}=\int_{V} \zeta(1-2 \nu) d V .
\end{gathered}
$$

Moreover, the external applied forces have been chosen to be those of a simple dynamic compression, i.e. $\mathbf{b}^{e x t}=(0,0,-b)^{T}$, with $b=b_{0}+b_{1} \sin (\omega t)$. With this assumption, it can be checked that the loads appearing in equations (5) are defined as $f_{0}=k b_{0}$ and $f_{1}=k b_{1}$ with $k=\int_{V} x_{3} d V$.

Some comments are needed at this point:

- One can easily estimate the equivalent mass $M$ by assuming that the volume mass density of concrete specimens is homogeneous (and known) while the displacement field depends linearly on reference configuration variables and on elongation.

- We assume that elastic nonlinearities are involved only in the evolution equation for the variable $\varphi$.

- The function $\tilde{k}_{1} \varphi+\tilde{k}_{2} \varphi^{2}+\tilde{k}_{3} \varphi^{3}$ is assumed to be monotonously increasing.

- The deformation energy clearly needs to be definite positive and consequently suitable restrictions on the stiffness parameters must be considered.

- The amplitude of the Coulombian frictional force, for obvious physical reasons, has to be smaller than the lowest amplitude of the applied external load (see the papers $[91,102,85]$ and references there cited for a discussion of the possible bifurcation phenomena which may occur).

- Many other possible dissipation regimes can be conceived in order to regularize the discontinuous dependence assumed in Coulombian model for friction: each of them would represent a different physical phenomenon, which would have different effects on the turning points of dissipation loops (i.e. when $|\dot{\varphi}|$ is suitably small). In this paper we assume that viscous Navier-Stokes dissipation effects are dominant when the microstructure velocity $|\dot{\varphi}|$ is suitably small, while they vanish and need to be replaced by Coulombian friction beyond a given micro-velocity level. 


\section{Numerical simulations: specimen in pure com- pression}

We limit our attention to numerical a model for a cylindrical specimen with diameter $\phi=11,28 \mathrm{~cm}$ and height $h=22 \mathrm{~cm}$. All simulations are performed via the automatic code COMSOL Multiphysics. As for boundary conditions, the specimen is constrained at the bottom with a zero displacement in its normal direction $x_{3}$. A cyclic external load per unit area is applied on the top with a frequency of $1 \mathrm{~Hz}$, very low compared with the natural frequencies of the testing sample to avoid obnoxious inertial effects. For the same reason, an initial smooth ramp is considered as showed in the figure 3 . In order to illustrate the

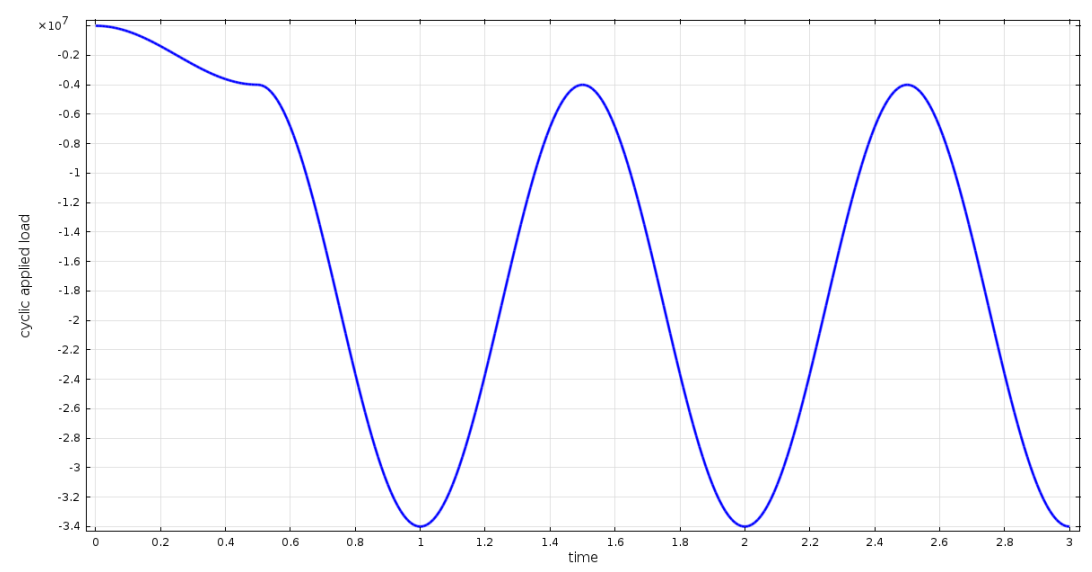

Figure 3: Cyclic external load

performances of the proposed model for the cement-based materials presented in this paper, we consider several numerical simulations performed by varying the parameters introduced in equation 5 . We show in table 2 the values of the basic parameters of our model used in the preformed numerical simulations.

\begin{tabular}{|c|c|c|}
\hline Parameter & Value & Unit \\
\hline \hline$M$ & $7.8 \times 10^{-2}$ & $\mathrm{~kg} \mathrm{~m}^{2}$ \\
\hline$m_{\varphi}$ & $7.8 \times 10^{-5}$ & $\mathrm{~kg}$ \\
\hline$K$ & $25 \times 10^{9}$ & $N m$ \\
\hline$\tilde{\alpha}$ & $1.9 \times 10^{11}$ & $N$ \\
\hline
\end{tabular}

\begin{tabular}{|c|c|c|}
\hline Parameter & Value & Unit \\
\hline \hline$\tilde{k}_{1}$ & $1.86 \times 10^{12}$ & $\mathrm{~N} / \mathrm{m}$ \\
\hline$\tilde{k}_{2}$ & $9.4 \times 10^{13}$ & $\mathrm{~N} / \mathrm{m}^{2}$ \\
\hline$\tilde{k}_{3}$ & $9.2 \times 10^{19}$ & $\mathrm{~N} / \mathrm{m}^{3}$ \\
\hline$\tilde{\zeta}$ & $1.5 \times 10^{11}$ & $\mathrm{~N}$ \\
\hline$\eta$ & $2 \times 10^{2}$ & $\mathrm{~s} / \mathrm{m}$ \\
\hline
\end{tabular}

Table 1: Reference values of the parameters used in the numerical simulations.

The numerical values shown in Tab. 1 have been determined by means of the following calibration process: 
- in the static loading cases the measured stress-strain relationship has to be verified;

- the value of the parameter $\varphi$ has to be compatible with the order of magnitude of known linear dimensions of typical cracks in concrete;

- equivalent mass coefficients are determined by taking into account volume mass density of concrete and an estimate of the percentage of total mass of concrete specimens which is moved because of crack lips movement;

- amplitude of Coulomb friction force has to be smaller than the force deforming micro-cracks;

- coupling between micro and macro motion must respect definite positiveness of deformation energy;

Some parametric studies are performed in the remainder of this paper on the crucial parameters of the presented model. Unless otherwise specified, the typical ranges of values assigned to the material parameters in the performed numerical simulations are those listed in table 2 .

\begin{tabular}{|c|c|c|}
\hline Parameter & Range of values & Unit \\
\hline \hline$\tilde{k}_{1}$ & $1.7 \times 10^{12}-1.93 \times 10^{12}$ & $N / m$ \\
\hline$\tilde{k}_{2}$ & $0-8 \times 10^{15}$ & $N / m^{2}$ \\
\hline$\tilde{k}_{3}$ & $6.5 \times 10^{19}-9.5 \times 10^{20}$ & $N / m^{3}$ \\
\hline$\tilde{\alpha}$ & $1.7 \times 10^{11}-2 \times 10^{11}$ & $N$ \\
\hline$\tilde{\zeta}$ & $10^{10}-4 \times 10^{11}$ & $N$ \\
\hline
\end{tabular}

Table 2: Ranges of values of the material parameters to be used in the numerical simulations on concrete.

\subsection{Parametric analysis of dissipation loops for varying friction coefficient}

In this subsection we consider the effect of the Coulombian friction coefficient $\tilde{\zeta}$ on the amplitude of dissipation loops, the area of which increases monotonically with $\tilde{\zeta}$. However, because of the strong non linearity of considered system, also the variation of the other relevant parameters is greatly influencing the dissipating capability as well. 


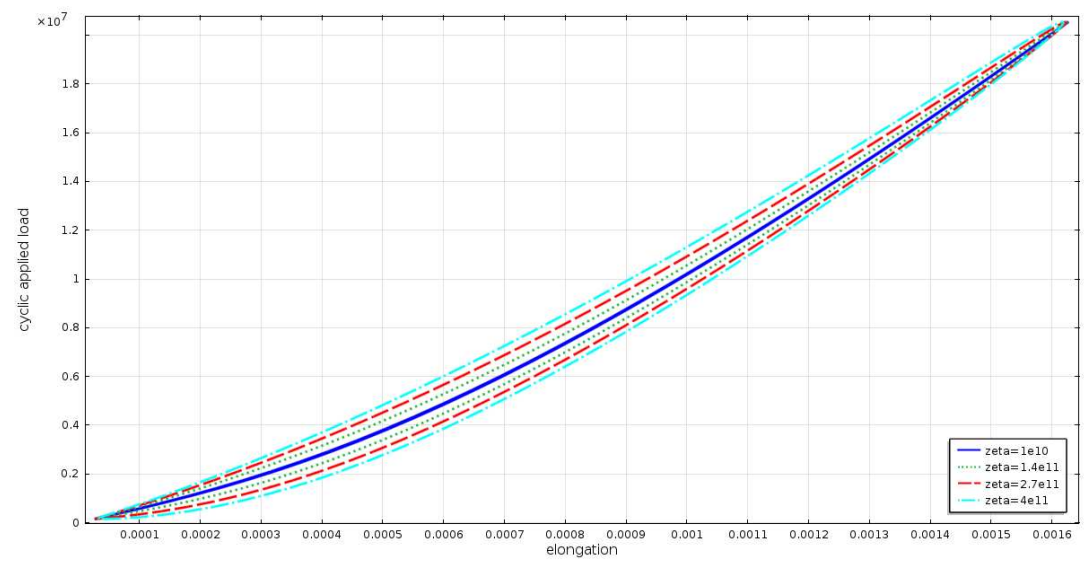

Figure 4: Influence of the friction coefficient $\tilde{\zeta}$ on the energy dissipation loops for cement-based materials. $\tilde{\zeta}=\left[1 \times 10^{10}, 1.4 \times 10^{11}, 2.710^{11}, 4 \times 10^{11}\right]$.

Figure 4 shows the variation of energy dissipation loops in a stress-strain diagram when varying the friction coefficient $\tilde{\zeta}$. It can be remarked that the area of the loops is increased when increasing the value of the coefficient $\tilde{\zeta}$. This is completely sensible, since the mechanism which we want to associate to the parameter $\tilde{\zeta}$ is the dissipation (at the scale of the micro-cracks) which is due to the relative motion of the two superimposed lips of each crack as a consequence of the application of the external dynamic load. This numerical evidence is a step towards the conception of suitable experimental campaigns on concrete modified with micro-fillers enhancing its dissipative properties.

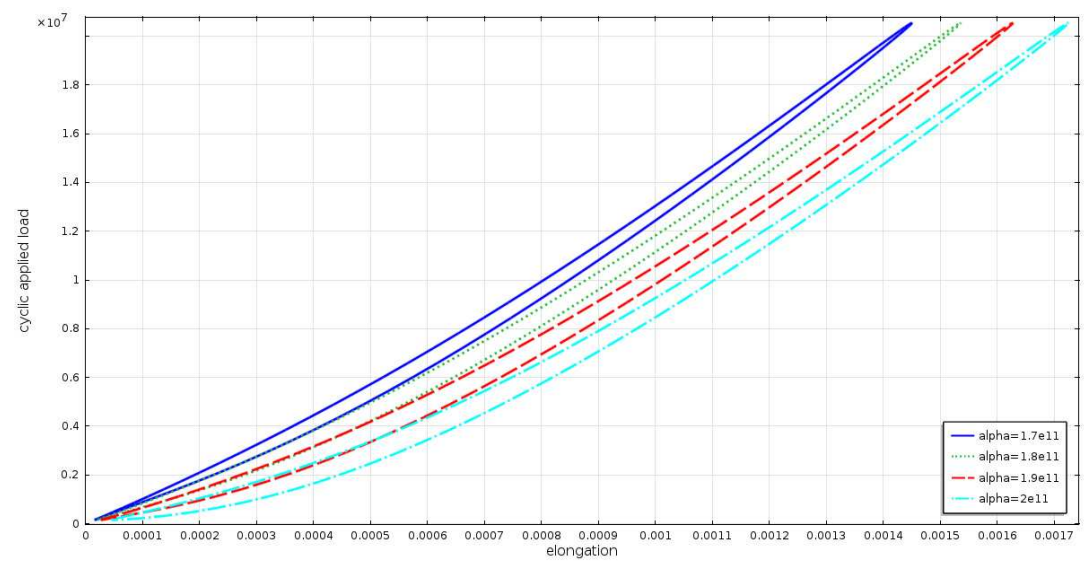

Figure 5: Influence of the material parameter $\tilde{\alpha}$ on the energy dissipation loops for cement-based materials. $\tilde{\alpha}=\left[1.7 \times 10^{11}, 1.8 \times 10^{11}, 1.9 \times 10^{11}, 2 \times 10^{11}\right]$. 
Figure 5 shows the variation of dissipation loops when varying the coefficient $\tilde{\alpha}$, i.e. the coupling parameter between the micro-structural variable $\varphi$ and the macroscopic strain. It is worth noticing that the effect of increasing micro-macro coupling implies that the dissipation loop is shifted towards the right. This means that the contribution to macroscopic deformation due to microscopic strain becomes more and more important and greater macroscopic strains can be attained with the same force level. Moreover, with a small coupling, the obtained loops can be seen to show a slightly smaller area and thus a reduced dissipation.

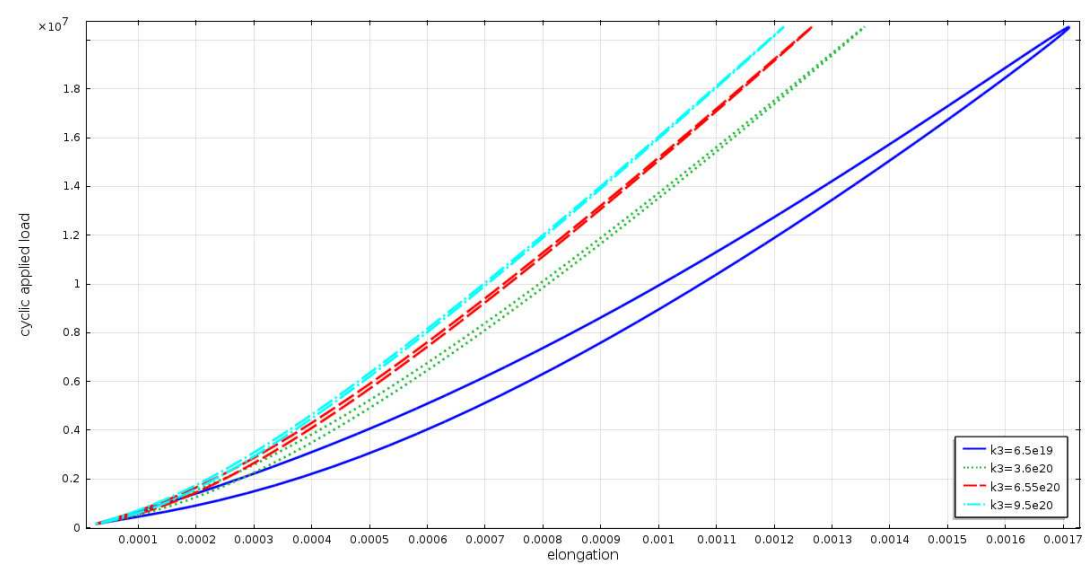

Figure 6: Influence of the material parameter $\tilde{k}_{3}$ on dissipation loops for cementbased materials. $\tilde{k}_{3}=\left[6.5 \times 10^{19}, 3.6 \times 10^{20}, 6.55 \times 10^{20}, 9.5 \times 10^{20}\right]$.

In figure 6 dissipation loops are depicted which show the effect of the material parameter $\tilde{k}_{3}$. It is possible to remark that, contrarily to what happens with the coupling parameter $\tilde{\alpha}$, high values of $\tilde{k}_{3}$ increase the whole stiffness of the system and consequently decrease the energy dissipated in each cycle. 


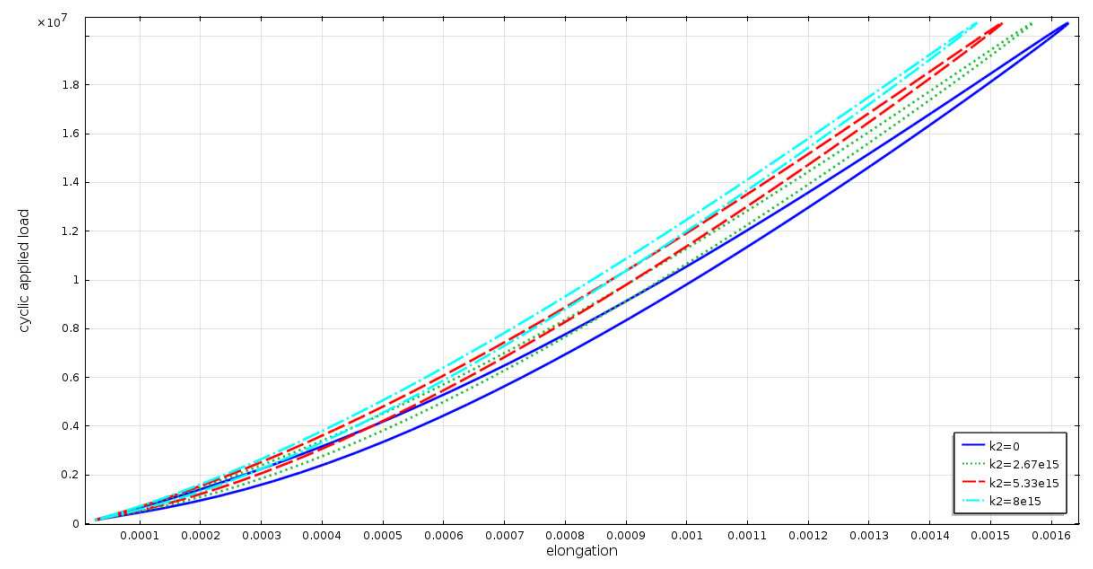

Figure 7: Influence of the material parameter $\tilde{k}_{2}$ on energy dissipation loops for cement-based materials. $k_{2}=\left[0,2.67 \times 10^{15}, 5.33 \times 10^{15}, 8 \times 10^{15}\right]$

In Fig. 7 the behavior of dissipation loops is depicted when increasing the value of the material parameter, $\tilde{k}_{2}$. A similar qualitative behaviour can be observed for the parameter $\tilde{k}_{2}$, even if the influence of this parameter is less pronounced when compared with that due to $\tilde{k}_{3}$. This is sensible since the microscopic non-linearities associated to the parameter $\tilde{k}_{3}$ are of higher order than those associated to $\tilde{k}_{2}$. We can hence conclude that the parameters $\tilde{k}_{2}$ and $\tilde{k}_{3}$ can be seen as averaged measures of the distribution of cracks inside the material: higher values of $\tilde{k}_{2}$ and $\tilde{k}_{3}$ can be associated to higher densities of cracks which allow for higher values of dissipation but somehow make the specimen softer than an equivalent one presenting lower densities of cracks.

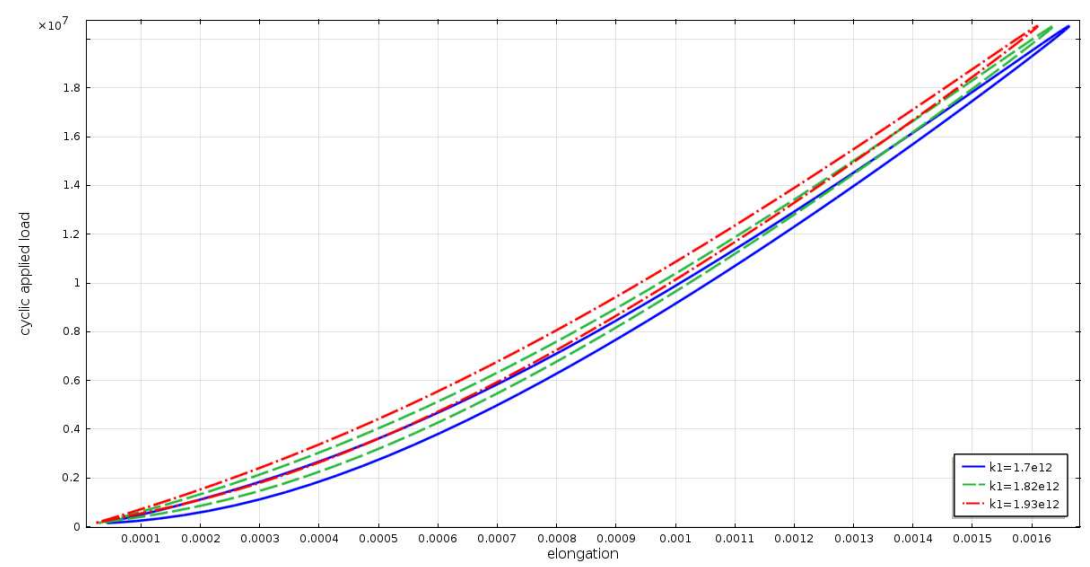

Figure 8: Computed energy dissipation loop for cement-based material - Influence of the material parameter $k_{1}\left(k_{1}=\left[1.710^{12}, 1.810^{12}, 1.9310^{12}\right]\right)$ 
Figure 8 shows how the material parameter $\tilde{k}_{1}$ affects the stress-strain cycles. It is possible to notice that an increase of the parameter $\tilde{k}_{1}$ do not directly affect the amount of energy dissipation, but changes the stiffness of the considered material.The coefficient $\tilde{k}_{1}$ can thus be seen as a coefficient directly related to the type of filler which is used to modify the concrete mix. Fillers with the same friction coefficient but with different mechanical properties could give rise to mechanical behaviors which can be encompassed by tuning the parameter $\tilde{k}_{1}$ alone.

\subsection{Some typical plots showing the periodic variation in time of relevant quantities}

It is well-known (see e.g. [145]) that well-behaving ODEs when forcing terms are periodic show solutions which are periodic as well. The dissipation effects which we have introduced in the previous equations 5 have been regularized in order to be sure that the aforementioned well-behaviour assumptions are verified. Therefore, when the simulation is performed for a time interval which is long enough, the calculated solution will become -within numerical errorperiodic: by a judicious choice of initial conditions the convergence towards stationary solutions has been reduced.

We plot the following time dependent quantities

- friction term in the equation for $\varphi$;

- the crack lips relative displacement $\varphi$ and its time derivative;

- the specimen elongation $\varepsilon$ and its time derivative.

Fig. 9 shows the typical periodic evolution for Coulomb friction. The different stiffness properties due to the introduced nonlinear term produces an asymmetric evolution of this friction term. As a consequence the relative dissipation loops will show a distance between the loading/unloading branches whose varying shape strongly depends on nonlinear behaviour considered. 


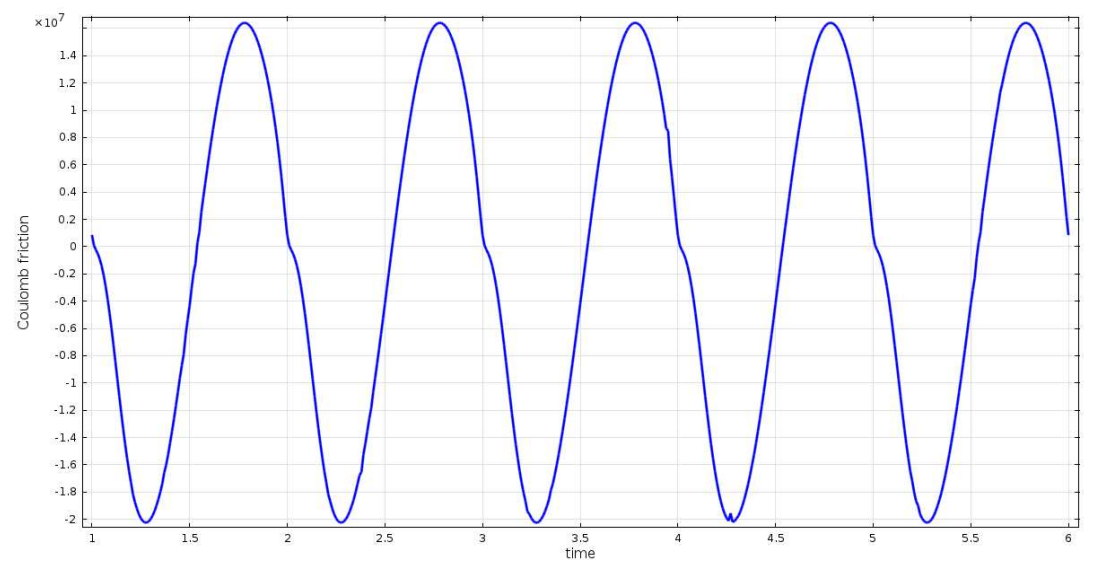

Figure 9: Periodic evolution for Coulomb friction

Fig. 10 shows the typical periodic evolution of the crack lips relative displacement. We notice a different behaviour in the neighborhood of the maximum and minimum level of the cyclic external compressive load due to elastic nonlinearities of the considered model. It is even worth noticing that near to aforementioned levels, two different time delays with respect to the applied cyclic load can be detected. This circumstance is related to the assumed dissipation mechanism and to the introduced elastic nonlinearities.

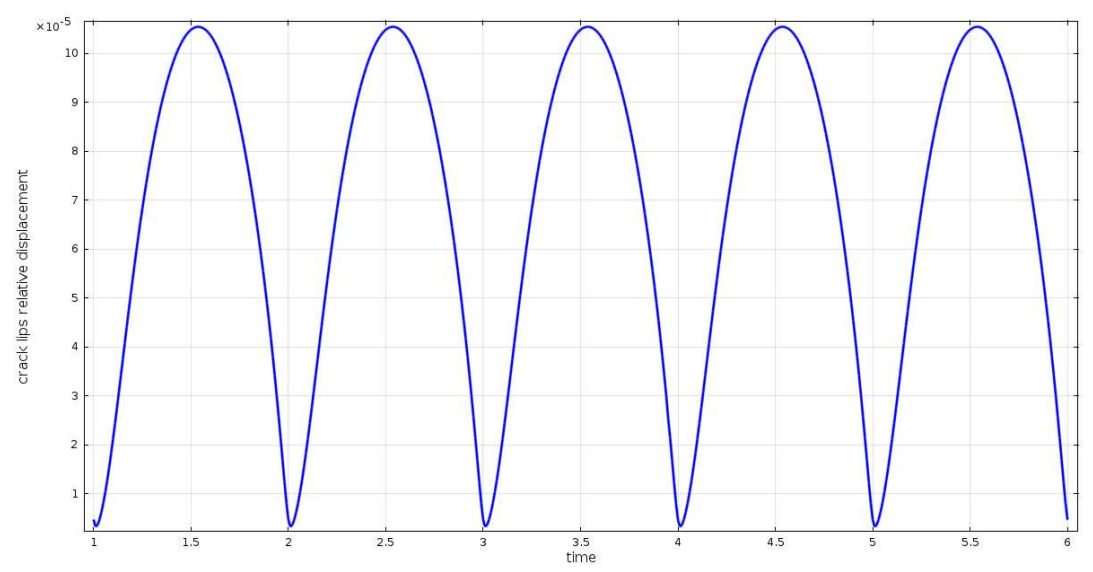

Figure 10: Periodic evolution of the crack lips relative displacement

Fig. 11 shows the typical periodic evolution of the specimen elongation. Similar considerations as those raised for the variable $\varphi$ can be made for the elongation, even if in this case the differences in the neighborhood of the maximum and minimum amplitude of the cyclic load are less emphasized. 


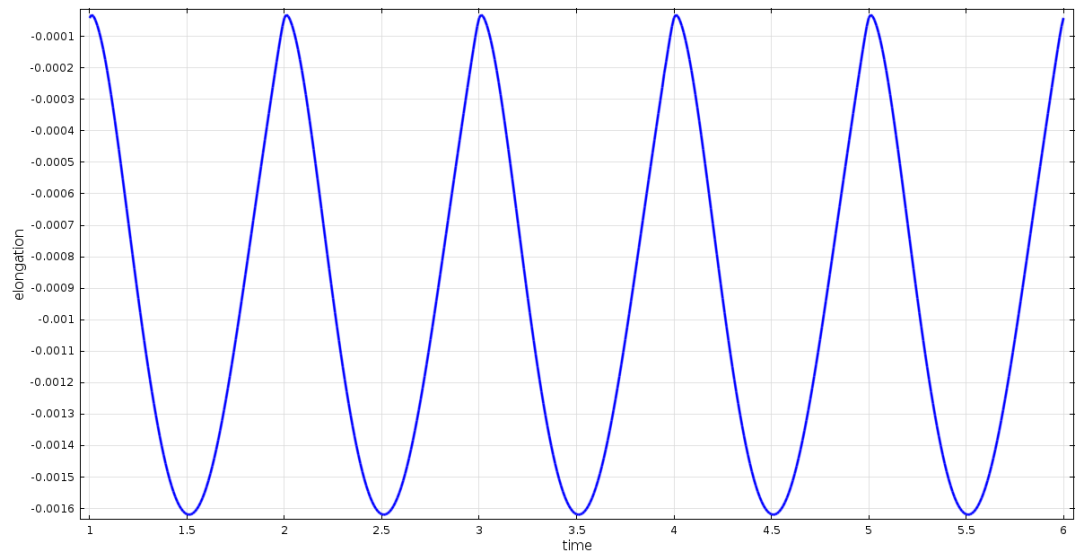

Figure 11: Periodic evolution of the specimen elongation

Fig. 12 shows the typical periodic evolution of the relative velocity of crack lips. The time dependence of this velocity shows clearly the effect of introduced Coulombian dissipation effect.

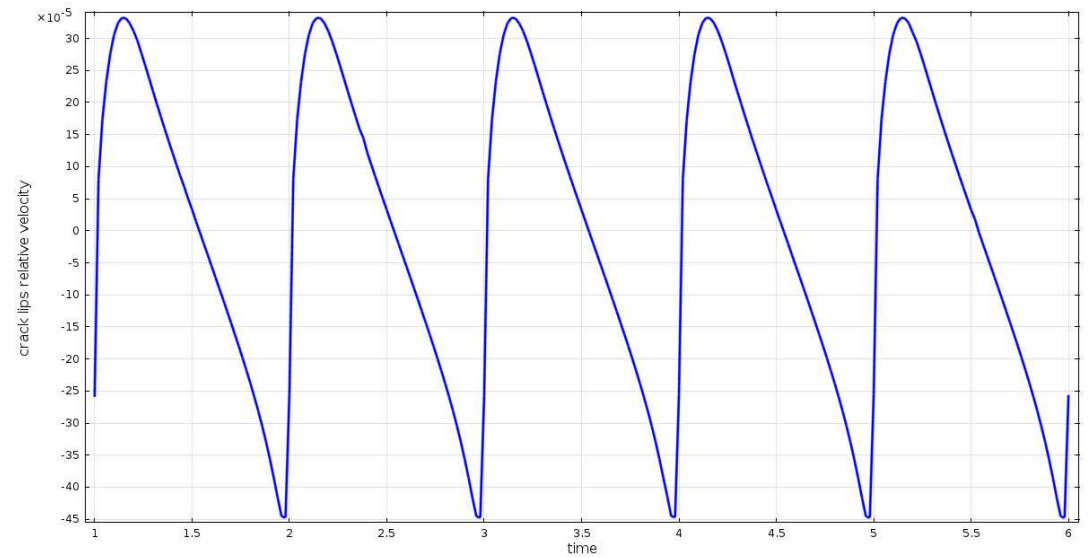

Figure 12: periodic evolution of crack lips relative velocity

Fig. 13 shows the typical periodic evolution of the specimen elongation rate. It is possible, also in these time evolution plots, to clearly detect the different effect of Coulombian dissipation in the loading/unloading cycles due to the introduced elastic nonlinearity. 


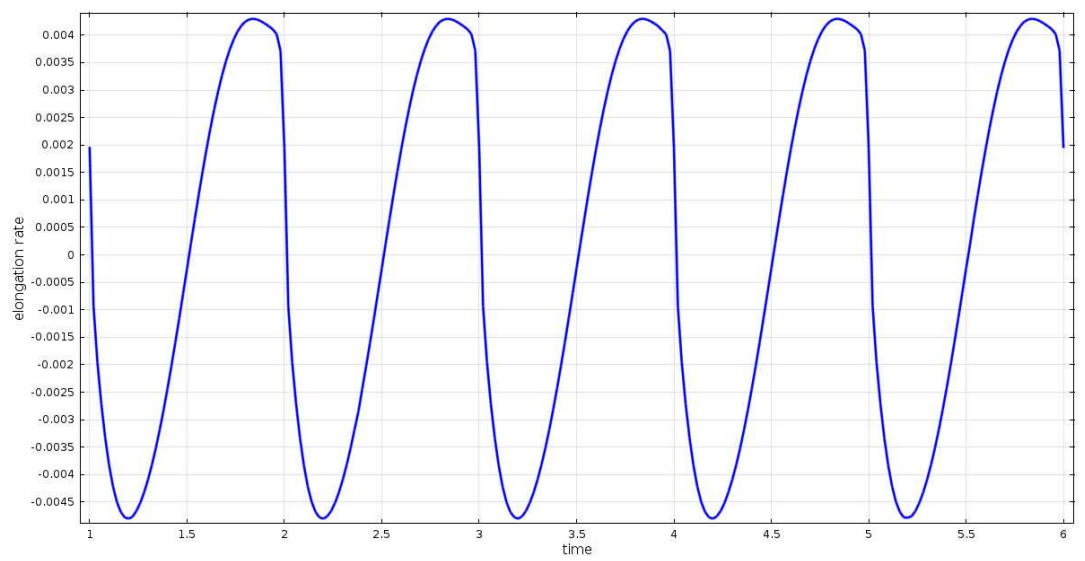

Figure 13: Periodic evolution of the specimen elongation rate

\section{Conclusions}

The modelling process presented in the present paper proves that it is possible, by means of a simple nonlinear system of ordinary differential equations, to carefully describe both quantitatively and qualitatively the available experimental evidence on compression of cylindrical modified concrete specimen. In particular, it is proven that, even if the applied compression force is related to elongation by a (nearly) linear relationship, some nonlinearities in the dissipation loop may be ascribed to nonlinear elastic phenomena involving the newly introduced microstructural parameter which describes internal micro-motions. More precisely, we want to interpret the physical meaning of this micro-structural parameter in terms of the overall relative displacement of the lips of the cracks which characterize the microstructure of concrete-type specimens.

The time rate of the same micro-structural parameter is the source of the only friction effect considered in this paper: the one related to Coulomb friction force.

By means of a series of numerical simulations, we have proved that a physically reasonable set of ranges for introduced constitutive parameters can be found which describes the whole variety of dissipation loops measured in [94].

The presented results justify the need for more complex models in order to describe i) bending and compression periodic deformation of considered cylindrical specimen, ii) coupling between bending and shear with micro-deformation iii) more complex micro-motions in which relative displacement of crack lips may depend on the orientation and localization of cracks iv) the longer range effects of micro-cracks-induced micro-deformation in the neighborhood of considered REV, i.e. those effects which can be modeled by allowing for the dependence of deformation energy on higher gradients of introduced kinematical fields. 


\section{References}

[1] L. Adelaide, B. Richard, F. Ragueneau, C. Cremona, Thermodynamical admissibility of a set of constitutive equations coupling elasticity, isotropic damage and internal sliding, C. R. Mecanique 338 (2010) 158-163.

[2] J.J. Alibert, P. Seppecher, F. dell'Isola, Truss modular beams with deformation energy depending on higher displacement gradients, Mathematics and Mechanics of Solids 8 (2003) 51-73.

[3] H. Altenbach, V.A. Eremeyev, Analysis of the viscoelastic behavior of plates made of functionally graded materials, ZAMM Zeitschrift fur Angewandte Mathematik und Mechanik, 88(5) (2008) 332-341.

[4] H. Altenbach, V.A. Eremeyev, On the bending of viscoelastic plates made of polymer foams, Acta Mechanica 204(3-4) (2009) 137-154.

[5] H. Altenbach, V.A. Eremeyev, On the linear theory of micropolar plates, ZAMM Zeitschrift fur Angewandte Mathematik und Mechanik 89(4) (2009) 242-256.

[6] H. Altenbach, V.A. Eremeyev, On the effective stiffness of plates made of hyperelastic materials with initial stresses, International Journal of NonLinear Mechanics 45(10) (2010) 976-981.

[7] H. Altenbach, V.A. Eremeyev, L.P. Lebedev, L.A. Rendón, Acceleration waves and ellipticity in thermoelastic micropolar media, Archive of Applied Mechanics 80(3) (2010) 217-227.

[8] H. Altenbach, V.A. Eremeyev, L.P. Lebedev, On the spectrum and stiffness of an elastic body with surface stresses, ZAMM Zeitschrift fur Angewandte Mathematik und Mechanik 91(9) (2011) 699-710.

[9] H. Altenbach, V.A. Eremeyev, On the shell theory on the nanoscale with surface stresses, International Journal of Engineering Science 49(12) (2011) 1294-1301.

[10] H. Altenbach, V.A. Eremeyev, N.F. Morozov, Surface viscoelasticity and effective properties of thin-walled structures at the nanoscale, International Journal of Engineering Science (2012) Article in Press.

[11] U. Andreaus, F. dell'Isola, M. Porfiri, Piezoelectric passive distributed controllers for beam flexural vibrations, JVC/Journal of Vibration and Control 10(5) (2004) 625-659.

[12] U. Andreaus, G.C. Ruta, A review of the problem of the shear centre(s) Continuum Mechanics and Thermodynamics 10 (1998).

[13] U. Andreaus, P. Baragatti, Fatigue crack growth, free vibrations, and breathing crack detection of aluminium alloy and steel beams, Journal of Strain Analysis for Engineering Design 44 (2009). 
[14] N. Auffray, R. Bouchet, Y. Brechet, Derivation of anisotropic matrix for bi-dimensional strain-gradient elasticity behaviour, International Journal of Solids and Structures 46(2) (2009) 440-454.

[15] N. Auffray, R. Bouchet, Y. Brechet, Strain gradient elastic homogenization of bidimensional cellular media, International Journal of Solids and Structures 47(13) (2010) 1698-1710.

[16] R.C. Batra, F. dell'Isola, G.C. Ruta, Second-order solution of SaintVenant's problem for an elastic bar predeformed in flexure, International Journal of Non-Linear Mechanics 40 (2-3) (2005) 411-422.

[17] A.O. Belyakov, A.P. Seyranian, A. Luongo, Dynamics of the pendulum with periodically varying length, Physica D: Nonlinear Phenomena 238 (16) (2009) 1589-1597.

[18] J. Beyrouthy, Reduction 3D-1D d'un modele viscoelastique en grandes deformations., C.R. Acad. Sci. Paris, Ser.I, Vol. 239-243 (2007) 115-130.

[19] J. Beyrouthy and P. Neff, A viscoelastic thin rod model for large deformations: numerical examples. Math. Mech. Solids, 16(8) (2011), 887-896.

[20] S.S. Bhattacharjee, P. Léger. Seismic cracking and energy dissipation in concrete gravity dams. Earthquake Engineering \& Structural Dynamics 22 (11) (1993) 991-1007.

[21] M. Bîrsan, H. Altenbach, T. Sadowski, V.A. Eremeyev, D. Pietras, Deformation analysis of functionally graded beams by the direct approach, Composites Part B: Engineering 43 (3) (2012) 1315-1328.

[22] M. Bongué Boma, M. Brocato. A continuum model of micro-cracks in concrete, Continuum Mechanics and Thermodynamics 22(2) (2010) 137161.

[23] Z. Boukria, A Limam, Experimental damage analysis of concrete structures using the vibration signature - Part II: Located damage (crack), International Journal of Mechanics 6 (1) (2012) 28-34.

[24] A.G. Bowland, R.E. Weyers, F.A. Charney, N.E. Dowling, T.M. Murray, and A. Ramniceanu, Effect of Vibration Amplitude on Concrete with Damping Additives. ACI Materials Journal, 109(3) (2012) 371-378.

[25] A. D. Brailsford, The role of internal stresses in internal friction, PhisicsLetter 8, 12(2) (1964).

[26] P.M. Buechner, R.S. Lakes, Size effects in the elasticity and viscoelasticity of bone, Biomechan. Model. Mechanobiol. 1 (2003) 295-301.

[27] A. Carcaterra, A. Akay, I.M. Ko, Near-irreversibility in a conservative linear structure with singularity points in its modal density, Journal of the Acoustical Society of America 119 (2006) 2141-2149. 
[28] A. Carcaterra, A. Akay, Theoretical foundations of apparent-damping phenomena and nearly irreversible energy exchange in linear conservative systems, Journal of the Acoustical Society of America 12 (2007) 19711982.

[29] A. Carcaterra, A. Akay, Dissipation in a finite-size bath, Physical Review E 84 (2011).

[30] A. Carpinteri, G. Lacidogna, N. Pugno, Scaling of energy dissipation in crushing and fragmentation: a fractal and statistical analysis based on particle size distribution, International Journal of Fracture 129 (2004) $131-139$.

[31] D. Ciancio, I. Carol, M. Cuomo, Crack opening conditions at 'corner nodes' in FE analysis with cracking along mesh lines, Engineering Fracture Mechanics 74 (13) (2007) 1963-1982.

[32] D. Ciancio, I. Carol, M. Cuomo, On inter-element forces in the FEMdisplacement formulation, and implications for stress recovery, International Journal for Numerical Methods in Engineering 66 (3) (2006) 502528.

[33] L. Contrafatto, M. Cuomo, A new thermodynamically consistent continuum model for hardening plasticity coupled with damage, International Journal of Solids and Structures 39 (2002) 6241-6271.

[34] L. Contrafatto, M. Cuomo, A framework of elastic-plastic damaging model for concrete under multiaxial stress states, International Journal of Plasticity 22 (2006) 2272-2300.

[35] L. Contrafatto, M. Cuomo, A globally convergent numerical algorithm for damaging elasto-plasticity based on the Multiplier method, International Journal for Numerical Methods in Engineering 63 (2005) 1089-1125.

[36] A. Culla, A. Sestieri, A. Carcaterra, Energy flow uncertainties in vibrating systems: Definition of a statistical confidence factor, Mechanical Systems and Signal Processing 17 (2003) 635-663.

[37] M. Cuomo, G. Ventura, Complementary Energy Approach to Contact Problems Based on Consistent Augmented Lagrangian regularization, Mathematical and Computer Modelling 28 (1998) 185-204.

[38] M. Cuomo, L. Contrafatto, Stress rate formulation for elastoplastic models with internal variables based on augmented Lagrangian regularisation, International Journal of Solids and Structures, 37 (2000) 3935-3964.

[39] F. Darve, S. Labanieh, Incremental constitutive law for sands and clays: simulations of monotonic and cyclic tests, International Journal for $\mathrm{Nu}$ merical and Analytical Methods in Geomechanics 6(2) (1982) 243-275. 
[40] F. dell'Isola, G. Ruta, and R. Batra, Generalized Poynting effects in predeformed prismatic bars, Journal of Elasticity 50 (1998) 181-196.

[41] F. dell'Isola, C. Woźniak, On phase transition layers in certain microdamaged two-phase solids, International Journal of Fracture 83 (2) (1997) $175-189$.

[42] F. dell'Isola, C. Woźniak, On continuum modelling the interphase layers in certain two-phase elastic solids, ZAMM Zeitschrift fur Angewandte Mathematik und Mechanik 77 (7) (1997) 519-526.

[43] F. dell'Isola, P. Seppecher, The relationship between edge contact forces, double force and interstitial working allowed by the principle of virtual power, Comptes rendus de l'Académie des Sciences Serie IIb 321 (1995) 303-308.

[44] F. dell'Isola, L. Rosa, L., C. Wozniak, Dynamics of solids with micro periodic nonconnected fluid inclusions, Archive of Applied Mechanics 67 (4) (1997) 215-228.

[45] F. dell'Isola, L. Rosa, L., C. Wozniak, A micro-structured continuum modelling compacting fluid-saturated grounds: The effects of pore-size scale parameter, Acta Mechanica 127 (1-4) (1998) 165-182.

[46] F. dell'Isola, K. Hutter, What are the dominant thermomechanical processes in the basal sediment layer of large ice sheets?, Proceedings of the Royal Society of London. Series A: Mathematical, Physical and Engineering Sciences 454 (1998) 1169-1195.

[47] F. dell'Isola, S. Vidoli, Damping of bending waves in truss beams by electrical transmission lines with PZT actuators, Archive of Applied Mechanics 68 (9) (1998) 626-636.

[48] F. dell'Isola, S. Vidoli, Continuum modelling of piezoelectromechanical truss beams: An application to vibration damping, Archive of Applied Mechanics 68 (1) (1998) 1-19.

[49] F. dell'Isola, R. Batra, Saint-Venant's Problem for Porous Linear Elastics Materials, Journal of Elasticity 47 (1997) 73-81.

[50] F. dell'Isola, M. Guarascio, K.A. Hutter, Variational approach for the deformation of a saturated porous solid. A second-gradient theory extending Terzaghi's effective stress principle, Archive of Applied Mechanics 70 (2000) 323-337.

[51] F. dell'Isola, A. Madeo, P. Seppecher, Boundary conditions at fluidpermeable interfaces in porous media: A variational approach, International Journal of Solids and Structures vol. 46(17) (2009) 3150-3164. 
[52] A. De Simone, A. Luongo, Nonlinear viscoelastic analysis of a cylindrical balloon squeezed between two rigid moving plates, International Journal of Solids and Structures 50 (2013) 2213-2223.

[53] A. Di Egidio, A. Luongo, A. Paolone, Linear and non-linear interactions between static and dynamic bifurcations of damped planar beams, International Journal of Non-Linear Mechanics 42 (1) (2007) 88-98.

[54] U.E. Shamy, C. Denissen, Microscale energy dissipation mechanisms in cyclically-loaded granular soils, Geotechnical and Geological Engineering 30(2) (2012) 343-361.

[55] V.A. Eremeyev, A.B. Freidin, L.L., Sharipova, Nonuniqueness and Stability in Problems of Equilibrium of Elastic Two-Phase Bodies, Doklady Physics 48 (7) (2003) 359-363.

[56] V.A. Eremeyev, W. Pietraszkiewicz, The nonlinear theory of elastic shells with phase transitions, Journal of Elasticity 74 (1) (2004) 67-86.

[57] V.A. Eremeyev, Acceleration waves in micropolar elastic media, Doklady Physics 50 (4) (2005) 204-206.

[58] V.A. Eremeyev, H. Altenbach, N.F. Morozov, The influence of surface tension on the effective stiffness of nanosize plates, Doklady Physics 54 (2) (2009) 98-100.

[59] V.A. Eremeyev, W. Pietraszkiewicz, Thermomechanics of shells undergoing phase transition, Journal of the Mechanics and Physics of Solids 59 (2011) 1395-1412.

[60] A.C. Eringen, Microcontinuum field theories, Springer-Verlag, New York, 2001.

[61] A.C. Eringen, E.S. Suhubi, Nonlinear theory of simple microelastic solids: I, Int. J. Eng. Sci. 2 (1964) 189-203.

[62] A.C. Eringen, E.S. Suhubi, Nonlinear theory of simple microelastic solids: II, Int. J. Eng. Sci. 2 (1964) 389-404.

[63] A.C. Eringen, D.G.B. Edelen, On nonlocal Elasticity, Int. J. Eng. Sci. 10 (1972) 233-248.

[64] A. C. Eringen, Microcontinuum Field Theories I. Foundations and Solids, Springer Verlag, 1999.

[65] G. Fantozzi and I.G. Ritchie, Internal friction caused by the intrinsic properties of dislocations, J. Phys. Colloques 42, C5-3-C5-23 (1981).

[66] G. Fantozzi, C. Esnouf, W. Benoit, I.G. Ritchie. Internal friction and microdeformation due to the intrinsic properties of dislocations: The Bordoni relaxation, Progress in Materials Science 27(3-4) (1982) 311-451. 
[67] S. Federico, W. Herzog, Towards an analytical model of soft biological tissues, Journal of Biomechanics 41(16) (2008) 3309-3313.

[68] S. Federico, T.C. Gasser, Nonlinear elasticity of biological tissues with statistical fibre orientation, Journal of the Royal Society Interface 7 (47) (2010) 955-966.

[69] S. Federico, W. Herzog, On the permeability of fibre-reinforced porous materials, International Journal of Solids and Structures 45 (7-8) (2008) $2160-2172$.

[70] M. Ferretti, A. Madeo, F. dell'Isola, P. Boisse, Modelling the onset of shear boundary layers in fibrous composite reinforcements by second gradient theory, ZAMP (2013) in press.

[71] S. Forest, R. Sievert, Nonlinear microstrain theories, Int. J. Solids Struct. 43 (2006) 7224-7245.

[72] S. Forest, Micromorphic Approach for Gradient Elasticity, Viscoplasticity, and Damage, Journal of Engineering Mechanics 135(3) (2009) 117-131.

[73] S. Forest, N.M. Cordero, E.P. Busso, First vs. second gradient of strain theory for capillarity effects in an elastic fluid at small length scales, Computational Materials Science 50 (2011) 1299-1304.

[74] Germain, P., 1972. Sur l'application de la méthode des puissances virtuelles en mécanique des milieux continus. Comptes Rendus De L'Academie Des Sciences 274, A1051-A1055.

[75] Germain, P., 1973. The method of virtual powers in continuum mechanics. Part two: Microstructure. Journal of Applied Mathematics 25 (3), $556-575$.

[76] Ghiba I.D., Neff P., Madeo A., Placidi L., Rosi G., 2013. The relaxed linear micromorphic continuum: existence, uniqueness and continuous dependence in dynamics. Submitted to Mathematics and Mechanics of Solids, arXiv:1308.3762v1 [math.AP]

[77] A.E. Green, R.S. Rivlin, Multipolar continuum mechanics, Archive for Rational Mechanics and Analysis 17(2) (1964) 113-114.

[78] A.E. Green, R.S. Rivlin, Simple force and stress multipoles, Archive for Rational Mechanics and Analysis 16, (1964) 325-353.

[79] A.E. Green, R.S. Rivlin, On Cauchy's equations of motion, Zeitschrift für Angewandte Mathematik und Physik (ZAMP) 15 (1964) 290-292.

[80] A.E. Green, R.S. Rivlin, Multipolar continuum mechanics: functional theory. I, Proceedings of the Royal Society of London. Series A: Mathematical, Physical and Engineering Sciences 284 (1965) 303-324. 
[81] F. Hernàndez-Olivares, G. Barluenga, M. Bollati, B. Witoszek, Static and dynamic behaviour of recycled tyre rubber-filled concrete, Cement and Concrete Research 32 (2002) 1587-1596.

[82] A. Hillerborg, M. Modéer, P.-E. Petersson, Analysis of crack formation and crack growth in concrete by means of fracture mechanics and finite elements, Cement and Concrete Research 6(6) (1976) 773-781.

[83] A. L. Kimball, D. E. Lovell, Internal Friction in Solids, Phys. Rev. 30 (1927) 948-959.

[84] P. Kotronis, R. Chambon, J. Mazars And F. Collin, Local second gradient models and damage mechanics: application to concrete, Bifurcations, Instabilities, Degradation in Geomechanics (2005) 127-142.

[85] M. Jamal, L. Lahlou, M. Midani, H. Zarouni, A. Limam, N. Damil, M. Potier-Ferry, A semi-analytical buckling analysis of imperfect cylindrical shells under axial compression, International Journal of Solids and Structures 40 (5) (2003) 1311-1327.

[86] L.P. Lebedev, M.J. Cloud, V.A. Eremeyev, Tensor Analysis with Applications in Mechanics, New Jersey: World Scientific (2010).

[87] T. Lekszycki, F. dell'Isola, A mixture model with evolving mass densities for describing synthesis and resorption phenomena in bones reconstructed with bio-resorbable materials, ZAMM - Journal of Applied Mathematics and Mechanics / Zeitschrift für Angewandte Mathematik und Mechanik (2012).

[88] C. Lomniz, Linear Dissipation in Solids, Journal of Applied Physics 28 (1957) 201-205.

[89] F. D'Annibale, A. Luongo, A damage constitutive model for sliding friction coupled to wear, Continuum Mechanics and Thermodynamics 25(2-4) (2013) 503-522.

[90] A. Luongo, Mode localization in dynamics and buckling of linear imperfect continuous structures, Nonlinear Dynamics, 25 (1-3) (2001) 133-156.

[91] A. Luongo, A. Di Egidio, Bifurcation equations through multiple-scales analysis for a continuous model of a planar beam, Nonlinear Dynamics 41 (2005) 171-190.

[92] A. Luongo, F. Romeo, A Transfer-matrix-perturbation approach to the dynamics of chains of nonlinear sliding beams, Journal of Vibration and Acoustics 128 (2006) 190-196.

[93] A. Luongo, D. Zulli, G. Piccardo, On the effect of twist angle on nonlinear galloping of suspended cables, Computers \& Structures 87 (2009) 10031014. 
[94] A. Madeo, Effect of Micro-Particle Additions on Frictional Energy Dissipation and Strength of Concrete University Libraries, Virginia Polytechnic Institute and State University, 2006.

[95] A. Madeo, T. Lekszycki, F. dell'Isola, A continuum model for the biomechanical interactions between living tissue and bio-resorbable graft after bone reconstructive surgery, Comptes Rendus - Mecanique 339 (10) (2011) 625-640.

[96] A. Madeo, D. George, T. Lekszycki, M. Nierenberger, Y. Rémond, A second gradient continuum model accounting for some effects of microstructure on reconstructed bone remodelling, Comptes Rendus Mécanique $340(8)$ (2012) $575-589$.

[97] A. Madeo, F. dell'Isola, F. Darve, A continuum model for deformable, second gradient porous media partially saturated with compressible fluids, Journal of the Mechanics and Physics of Solids 61 (2013) 2196-2211.

[98] C. Maurini, F. dell'Isola, D. del Vescovo, Comparison of piezoelectronic networks acting as distributed vibration absorbers, Mechanical Systems and Signal Processing 18 (2004) 1243-1271.

[99] C. Maurini, J. Pouget, F. dell'Isola, On a model of layered piezoelectric beams including transverse stress effect, International journal of solids and structures 41 (2004) 4473?4502.

[100] C. Maurini, J. Pouget, F. dell?Isola, Extension of the Euler Bernoulli model of piezoelectric laminates to include 3D effects via a mixed approach, Computers and Structures 84 (2006) 1438-1458.

[101] P. K. Mehta, Paulo J. M. Monteiro, Concrete: Microstructure, Properties, and Materials, McGraw-Hill, 2005.

[102] G. Michel, A. Limam, J.F. Jullien, Buckling of cylindrical shells under static and dynamic shear loading, Engineering Structures 22 (5) (2000) $535-543$.

[103] R.D. Mindlin, Micro-structure in linear elasticity, Archive for Rational Mechanics and Analysis 16 (1964) 51-78.

[104] R.D. Mindlin, Second gradient of strain and surface tension in linear elasticity, International Journal of Solids and Structures 1 (1965) 417-438.

[105] R.D. Mindlin, N.N. Eshel, On first strain-gradient theories in linear elasticity, International Journal of Solids and Structures, 4 (1968) 109-124.

[106] A. Misra, C.S. Chang, Effective Elastic Moduli of Heterogeneous Granular Solids, International Journal of Solids and Structures, 30 (1993) 25472566 . 
[107] A. Misra, Y. Yang, Micromechanical model for cohesive materials based upon pseudo-granular structure, International Journal of Solids and Structures 47 (2010) 2970-2981.

[108] A. Misra, V. Singh, Micromechanical model for viscoelastic-materials undergoing damage, Continuum Mechanics and Thermodynamics, 25 (2013) $1-16$.

[109] A. Misra, W.Y. Ching, Theoretical nonlinear response of complex single crystal under multi-axial tensile loading, Scientific Reports, 3 (2013).

[110] B. Nadler, P. Papadopoulos, D.J. Steigmann, Multiscale constitutive modeling and numerical simulation of fabric material, International Journal of Solids and Structures, 43 (2006) 206-221.

[111] Neff P., Ghiba I.D., Madeo A., Placidi L., Rosi G., 2013. A unifying perspective: the relaxed micromorphic continuum. Existence, uniqueness and continuous dependence in dynamics. Submitted to Continuum Mechanics and Thermodynamics, arXiv:1308.3219v1 [math-ph]

[112] P. Neff, Finite multiplicative plasticity for small elastic strains with linear balance equations and grain boundary relaxation. Cont. Mech. Thermodynamics, 15(2) (2003) 161-195.

[113] P. Neff, Local existence and uniqueness for quasistatic finite plasticity with grain boundary relaxation. Quart. Appl. Math., 63 (2005) 88-116.

[114] P. Neff, A finite-strain elastic-plastic Cosserat theory for polycrystals with grain rotations. Int. J. Eng. Sci., 44 (2006) 574-594.

[115] P. Neff, A geometrically exact viscoplastic membrane-shell with viscoelastic transverse shear resistance avoiding degeneracy in the thin-shell limit. Part I: The viscoelastic membrane-plate. Zeitschrift Angewandte Mathematik Physik (ZAMP), 56(1) 2005 148-182.

[116] P. Neff, Local existence and uniqueness for a geometrically exact membrane-plate with viscoelastic transverse shear resistance. Math. Meth. Appl. Sci., 28 (2005) 1031-1060.

[117] V. Pensee, D. Kondo, L. Dormieux, Micromechanics of anisotropic damage in rocks and concrete: unilateral effects modeling and coupling with friction, 15th ASCE Eng. Mechanics Conf. 2002.

[118] W. Pietraszkiewicz, V.A. Eremeyev, On vectorially parameterized natural strain measures of the non-linear Cosserat continuum, International Journal of Solids and Structures 46 (11-12) (2009) 2477-2480.

[119] W. Pietraszkiewicz, V.A. Eremeyev, V. Konopinska, Extended non-linear relations of elastic shells undergoing phase transitions, Zeitschrift für Angewandte Mathematik und Mechanik (ZAMM) 87 (2007) 150-159. 
[120] J. Pohd, O.T. Bruhns, On a thermodynamical consistent constitutive law based on the concept of internal variables, Mechanics Research Communications 20(2) (1993) 99-105.

[121] M. Porfiri, F. dell'Isola, E. Santini, Modeling and design of passive electric networks interconnecting piezoelectric transducers for distributed vibration control, International Journal of Applied Electromagnetics and Mechanics 21 (2) (2005) 69-87.

[122] S. Quiligotti, G.A. Maugin, F. dell'Isola, An Eshelbian approach to the nonlinear mechanics of constrained solid-fluid mixtures, Acta Mechanica 160 (2003) 45-60.

[123] S. Quiligotti, G. A. Maugin, F. dell'Isola, Wave motions in unbounded poroelastic solids infused with compressible fluids, Zeitschrift für angewandte Mathematik und Physik ZAMP 53(6) (2002) 1110-1138.

[124] A. Rinaldi, Y.C. Lai, Damage Theory Of 2D Disordered Lattices: Energetics And Physical Foundations Of Damage Parameter, Int. J. Plasticity 23 (2007) 1796-1825.

[125] A. Rinaldi, D. Krajcinovic, S. Mastilovic, Statistical Damage Mechanics and Extreme Value Theory, Int. J. Damage Mech, 16(1) (2007) 57-76.

[126] A. Rinaldi, Y.C. Lai, Statistical damage theory of 2D lattices: Energetics and physical foundations of damage parameter, International Journal of Plasticity 23 (2007) 1796-1825.

[127] A. Rinaldi, D. Krajcinovic, P. Peralta, Y.C. Lai, Lattice models of polycrystalline microstructures: A quantitative approach. Mechanics of Materials 40 (2008) $17-36$.

[128] A. Rinaldi, A rational model for 2D Disordered Lattices Under Uniaxial Loading, Int. J. Damage Mech. 18 (2009) 233-57.

[129] A. Rinaldi, Statistical model with two order parameters for ductile and soft fiber bundles in nanoscience and biomaterials, Phys Rev E 83(4-2) (2011).

[130] A. Rinaldi, Bottom-up modeling of damage in heterogeneous quasi-brittle solids, Continuum Mechanics and Thermodynamics 25(2-4) (2013) 359373 .

[131] J. C. Savage, J. D. Byerlee, and D. A. Lockner. Is internal friction friction?, Geophysical research letters 23(5) (1996) 487-490.

[132] C. J. Spears, P. Feltham, On the amplitude-independent internal friction in crystalline solids, Journal of Materials Science 7(8) (1972) 969-971. 
[133] G. Sciarra, F. dell'Isola, K. Hutter, A solid-fluid mixture model allowing for solid dilatation under external pressure, Continuum Mechanics and Thermodynamics 13(5) (2001) 287-306.

[134] G. Sciarra, F. dell'Isola, O. Coussy, Second gradient poromechanics, Int. J. Solids Struct 44(20) (2007) 6607-6629.

[135] L.I. Sedov, Models of continuous media with internal degrees of freedom, Journal of Applied Mathematics and Mechanics 32 (1972) 803-819.

[136] R.A. Toupin, Elastic Materials with couple-stresses, Archive for Rational Mechanics and Analysis 11 (1962) 385-414.

[137] R.A. Toupin, Theories of elasticity with couple-stress, Archive for Rational Mechanics and Analysis 17 (1964) 85-112.

[138] Y. Yang, A. Misra, Higher-order stress-strain theory for damage modeling implemented in an element-free Galerkin formulation, Computer Modeling in Engineering and Sciences 64 (2010) 1-36.

[139] Y. Yang, A. Misra, Micromechanics based second gradient continuum theory for shear band modeling in cohesive granular materials following damage elasticity, International Journal of Solids and Structures 49 (2012) $2500-2514$.

[140] Y. Yang, W.Y. Ching, A. Misra, Higher-order continuum theory applied to fracture simulation of nano-scale intergranular glassy film, Journal of Nanomechanics and Micromechanics 1 (2011) 60-71.

[141] C.-T. Yu, A.S. Kobayashi, N.M. Hawkins, Energy-dissipation Mechanisms Associated with Rapid Fracture of Concrete, Experimental Mechanics 33(3) (1993) 205-211.

[142] V.A. Yeremeyev, A.B. Freidin, L.L. Sharipova, The stability of the equilibrium of two-phase elastic solids, Journal of Applied Mathematics and mechanics (PMM) 71 (2007) 61-84.

[143] S. Vidoli, F. dell'Isola, Modal coupling in one-dimensional electromechanical structured continua, Acta Mechanica 141 (2000) 37-50.

[144] S. Vidoli, F. dell'Isola, Vibration control in plates by uniformly distributed PZT actuators interconnected via electric networks, European Journal of Mechanics, A/Solids 20(3) (2001) 435-456.

[145] M. Volpato, Sull'esistenza di soluzioni periodiche per equazioni differenziali ordinarie del secondo ordine. Rendiconti del Seminario Matematico della Università di Padova 25 (1956) 371-385.

[146] C. Zener, Internal friction in solids, Proc. Phys. Soc. 52 (1940) 152-166. 
[147] R. L. Wegel, H. Walther, Internal dissipation in solids for small cyclic strains, Physics 6 (1935) 141-157.

[148] K. Weinberg and P. Neff, A geometrically exact thin membrane modelinvestigation of large deformations and wrinkling. Int. J. Num. Meth. Engrg., 74(6) (2007) 871-893. 